\title{
Analysis and evaluation of probabilistic routing protocol for intermittently connected network
}

\author{
Jahnabi Borah $^{1}$, Dhruwajita Devi ${ }^{2}$, Dr. Y. Jayanta Singh ${ }^{3}$ \\ Dept of Comp. Sc Engg \& IT, Don Bosco College of Engg. \& Technology, Assam Don Bosco University,
} Assam.

\begin{abstract}
Intermittently connected network often referred to as Delay/Disruption Tolerant Network which is an infrastructure less network suffers from intermittent connection i.e. a connected path from the source to the destination may be absent or a given destination may not be reachable at the moment a message is sent. As a result traditional routing protocols for Ad hoc networks cannot serve the purpose of this type of intermittently connected network. PROPHET routing protocol works on the principle of history of encounters and transitivity. It is called Probabilistic routing protocol since it uses a parameter 'Delivery of Predictability'. In this paper the architecture of delay tolerant network is being discussed and PROPHET protocol is implemented.
\end{abstract}

Keywords: Delay tolerant network, PROPHET routing protocol, Delivery predictability, Epidemic routing protocol.

\section{Introduction}

Wireless mobile devices may establish communication at any moment and in any place, regardless of the existence of an end-to-end path between source and destination. In such environments, the occurrence of faults or the mobility pattern of some devices can cause network partitions, creating highly-partitioned networks. One way of communicating in such intermittently connected network is by allowing messages to be buffered at intermediate nodes for a longer time than normally occurs in the queues of conventional routers[4]. But this makes the network to consume more resources and it maximizes the delay in message delivery.

\subsection{Delay Tolerant Network}

Delay and disruption tolerant networks are occasionally-connected networks that may suffer from partitions frequently. Delay Tolerant Networks (DTN) is a new network architecture, which was first proposed by Kevin Fall in SIGCOMM 2003[6][1].

Networks which falls under the category of DTN include[2]:

- Interplanetary Internet, which focused primarily on the issue of deep space communication in high delay environments.

- Sensor-based networks using scheduled intermittent connectivity.

- Terrestrial wireless networks that cannot ordinarily maintain end-toend connectivity.

- Satellite networks with moderate delays and periodic connectivity, and

- Underwater acoustic networks with moderate delays and frequent interruptions due to environmental factors.

The traditional Internet protocols assume the following[2]:

- An end-to-end path between source and destination exists for the duration of a communication session (for reliable communication)

- Retransmissions based on timely and stable feedback from data receivers is an effective means for repairing errors

- end-to-end loss is relatively small

- All routers and end stations support the TCP/IP protocols

- Applications need not worry about communication performance

- endpoint-based security mechanisms are sufficient for meeting most security concerns

- Packet switching is the most appropriate abstraction for interoperability and performance

- Selecting a single route between sender and receiver is sufficient for achieving acceptable communication performance.

But the DTN architecture does not follow most of the above assumptions as a result traditional Internet Protocols cannot be applied to DTN. 
Some of the design principles which the DTN architecture prefers are summarized below:

- Storage within the network: When an end to end path between the source and the destination does not exist, the intermediate nodes should support store-and-forward operation over potentially long timescales. This type of network do not require end-to-end reliability.

- Security mechanisms should be there to protect it from unauthorized use by minimizing traffic as soon as possible.

- Variable-length (possibly long) messages (not streams or limited-sized packets)is preferred to be used as the communication abstraction to help enhance the ability of the network to make good scheduling/path selection decisions when possible.[2]

\subsection{Routing Mechanism in DTN}

Routing in DTN is totally different from routing in MANETS because there is frequent disconnections. So while designing a routing protocol for DTN, we have to take into consideration the following characteristics of DTN such as[3][5]:

- Intermittent connection

- Energy Constraint

- Delay in message delivery

- Long queuing of message

- Limitation of resource

- Limited Longevity

- Security

The DTN architecture defines routes to be computed based on a collection of "contacts" indicating the start time, duration, endpoints, forwarding capacity, and latency of a link in the topology graph [4]. The contacts may be "opportunistic" or "predicted" type.

Opportunistic contacts: They are not scheduled, but are encountered unexpectedly and this takes place frequently due to node mobility.

Predicted contacts: They are similar to opportunistic contacts, but they are based on some information. It can be possible to draw some statistical conclusion as to whether or not a contact will be present soon [4].

The Delay tolerant network also introduces the bundle protocol, by which applications can "bundle" both data and metadata, into a single message, or bundle, that can be sent as a unit. These units are of arbitrary length are called Application Data Units or ADUs. A node in a DTN sends and receives bundles("blocks" of data).

\subsection{Routing protocol for DTN}

Many routing protocols have been proposed for DTNs[7], such as

A. Deterministic Routing Protocols

Oracle based, Link state based, Space time based.[7]

B. Stochastic Routing

B.1 Passive Routing

Epidemic routing [8], PROPHET [5], Spray Wait [9] and many more.

\section{B.2 Active Routing}

Meet and Visit, Message Ferrying.[7]

\subsection{Epidemic Protocol}

In Epidemic Routing [8] messages are distributed to hosts or intermediate nodes between source and the destination, called carriers, within connected portions of ad hoc networks. This makes the messages to get quickly distributed through connected portions of the network. Then these carriers come into contact with another connected portion of the network through node's movement. In this way, the message spreads to a different network of nodes. Through such transitive transmission of data, messages have a high probability of eventually reaching their destination[8].

Say if , a source, $\mathrm{S}$, wishes to send a message to a destination, D ,but no connected path is available from $\mathrm{S}$ to $\mathrm{D}$, then $\mathrm{S}$ transmits its messages to its two neighbors, $\mathrm{C} 1$ and $\mathrm{C} 2$, within direct communication 
range. At some later time, $\mathrm{C} 2$ comes into direct communication range with another host, $\mathrm{C} 3$, and transmits the message to it. C3 is in direct range of D and finally sends the message to its destination. Epidemic Routing supports the eventual delivery of messages to their destinations with minimal assumptions regarding the underlying topology and connectivity of the underlying network[8]. Only periodic pair-wise connectivity is the means of ensuring eventual message delivery

\subsection{PROPHET Protocol}

PROPHET is somewhat different from the epidemic routing protocol for intermittently connected networks. It operates by pruning the distribution tree unlike the epidemic protocol and tries to minimize the usage of resource while still attempting to achieve the best-case routing capabilities. It is intended for use in sparse mesh networks where a fully connected path between the source and destination may or may not be present. The PROPHET routing protocol is preferable at situations with some of mobile nodes that creates connectivity patterns that are not completely random but can be predict. Human carrying mobile wireless devices create such mobility patterns by daily or weekly periodic activities.

In PROPHET protocol, nodes are expected to be able to establish a TCP link for exchanging the information. PROPHET uses a mechanism that is similar to the mechanism used in metric-based vector routing protocols where the metric might be distance or cost[4]. PROPHET is a Probabilistic Routing Protocol that uses History of Encounters and Transitivity[6]. In [6] they introduced a metric called "delivery predictability", $0<=\mathrm{P}_{-}(\mathrm{A}, \mathrm{B})<=1$, is established at every node A for each known destination $\mathrm{B}$. In PROPHET a node which has a higher delivery predictability value for a particular destination is assumed to be a better router for delivering a message to that destination (i.e., if $\mathrm{P}(\mathrm{A}, \mathrm{B})>\mathrm{P}(\mathrm{C}, \mathrm{B})$, message for destination $\mathrm{B}$ are preferable to forward to A rather than $\mathrm{C}[3]$.

Whenever two nodes implementing PROPHET protocol gets the opportunity to communicate, they enter a two-part Information Exchange Phase (IEP).

In the first part, the delivery predictabilities for all destinations known by each node are exchanged with the encountered node.

In the second part, this shared information is used by each node to update their internal delivery predictability. After that, the nodes exchange information such as :destination and size about the bundles each node carries, and these information is used in addition to the updated delivery predictabilities to decide which bundles to request to be forwarded from the other node. In the Information Exchange Phase the bundles are forwarded.

If node $\mathrm{B}$ has not met node $\mathrm{A}$ for a long time or has never met node $\mathrm{B}$, such that $\mathrm{P}(\mathrm{A}, \mathrm{B})<\mathrm{P} \_$threshold, then P_(A,B) should be set to $\mathrm{P}$ init. $\mathrm{P}$ init should be set to $0.5[3][5]$.

The calculation of the delivery predictability is done in three steps[3][5]:

- When a node A meets another node B: A updates the probability of delivery as shown in the following equation:

$\mathbf{P}(\mathrm{A}, \mathrm{B})=\mathbf{P}(\mathbf{A}, \mathrm{B})$ old $+(\mathbf{1}-\partial-\mathbf{P}(\mathbf{A}, \mathrm{B})$ old $) * \mathbf{P}$ init

The value of $\partial$ should normally be very small (e.g., 0.01).

- For a node $\mathrm{C}$ known by a node B: A updates the probability of delivery in accordance with the following equation:

$\mathbf{P}(\mathbf{A}, \mathbf{C})=\max (\mathbf{P}(\mathbf{A}, \mathbf{C})$ old, $\mathbf{P}(\mathbf{A}, \mathbf{B}) * \mathbf{P}(\mathbf{B}, \mathbf{C}) * \beta)$ where $0<=\beta<=1$ is a constant that determines the impact of transitivity on the delivery predictability.

- For nodes infrequently met by A: A updates the probability of delivery in accordance with the following equation:

$\mathbf{P}(\mathbf{A}, \mathbf{B})=\mathbf{P}(\mathbf{A}, \mathbf{B}) * \gamma^{\wedge} \mathbf{k}$ where $\gamma$ is an aging constant and and $\mathrm{k}$ is the number of time units that have elapsed since the last time the metric was aged.

There are some standard delivery predictability (dp) values[4]:

$\begin{array}{ll}\text { - P init } & 0.5 \\ \text { - P_threshold } & 0.1 \\ \text { - P_encounter max: } & 0.7 \\ \text { - alpha } & 0.5 \\ \text { - beta } & 0.9 \\ \text { - gamma } & 0.999\end{array}$


- delta

\subsection{Simulator setup}

0.01

\section{Performance Evaluation}

We conducted experiments and evaluated the performance of the PROPHET protocol using ns-2 packet-level simulator.100 mobile nodes were moved according to the random waypoint mobility model within a $500 \mathrm{~m} * 500 \mathrm{~m}$ area. Each node had a radio propagation range of $250 \mathrm{~m}$ and channel capacity was $2 \mathrm{Mb} / \mathrm{s}$. All simulations were run for 500 seconds of simulated time. We did our experiments with movement patterns for different pause times: 0, 100, 200, 300, 400 and 500 seconds. Data traffic was generated using constant bit rate(CBR) UDP traffic sources. Simulation study was performed for packet delivery ratio and energy consumed.

Delivery Ratio: When the message's destination has received the message, then that message will be called delivered message. The ratio between the no. of messages sent to the no. of message successfully received is the packet delivery ratio[3].

Energy spent: Since all the mobile wireless devices are battery powered, thus it is the battery capacity or the energy value that is spent by the nodes during the simulation time.

\subsection{Parameter settings}

The simulation parameters that have been used in our experiments are stated in figure 1

\begin{tabular}{|l|l|}
\hline Parameters & Value \\
\hline Simulation area & $500 \mathrm{X} 500 \mathrm{~m}$ \\
\hline Simulation time & $100 \mathrm{~s}$ \\
\hline energy & 40 watts \\
\hline No. of nodes & 100 \\
\hline Packet size & $512 \mathrm{~kb}$ \\
\hline Initial energy & 40 watts \\
\hline Rate & 250 kbps \\
\hline Max packet in ifq & 200 \\
\hline Initial transmit power & 0.660 \\
\hline Initial receive power & 0.395 \\
\hline Idle power & 0.035 \\
\hline P init & 0.5 \\
\hline P_threshold & 0.1 \\
\hline P_encounter max & 0.7 \\
\hline alpha & 0.5 \\
\hline beta & 0.9 \\
\hline gamma & 0.999 \\
\hline delta & 0.01 \\
\hline
\end{tabular}

Fig. 1 Table-Main Parameter Settings

\section{Result}

In the simulated experiment, for the first scenario we have focused on comparing the performance with regard to two metrics: average packet delivery rates and energy spent with respect to simulation time o sec to 100 secs. 


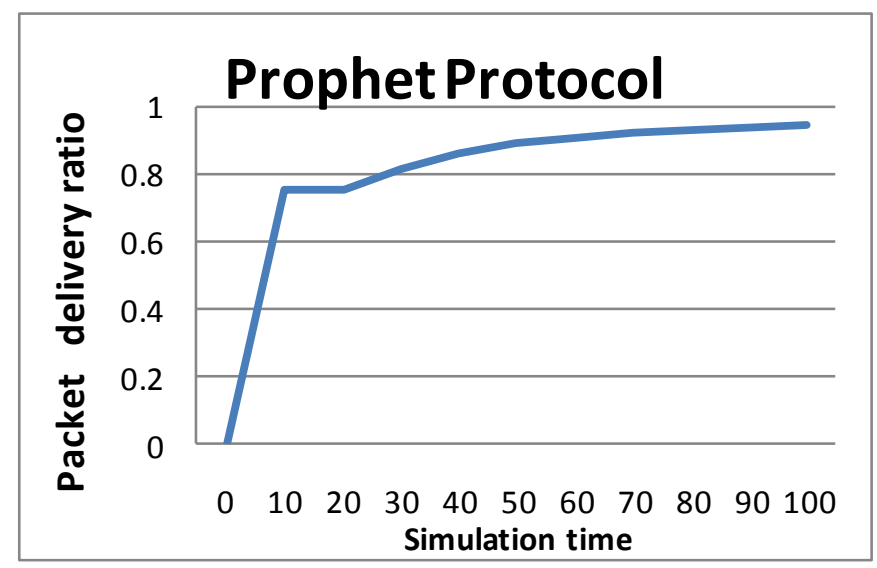

Fig. 2 shows average packet delivery rates as the time increases, the average PDR also increases.

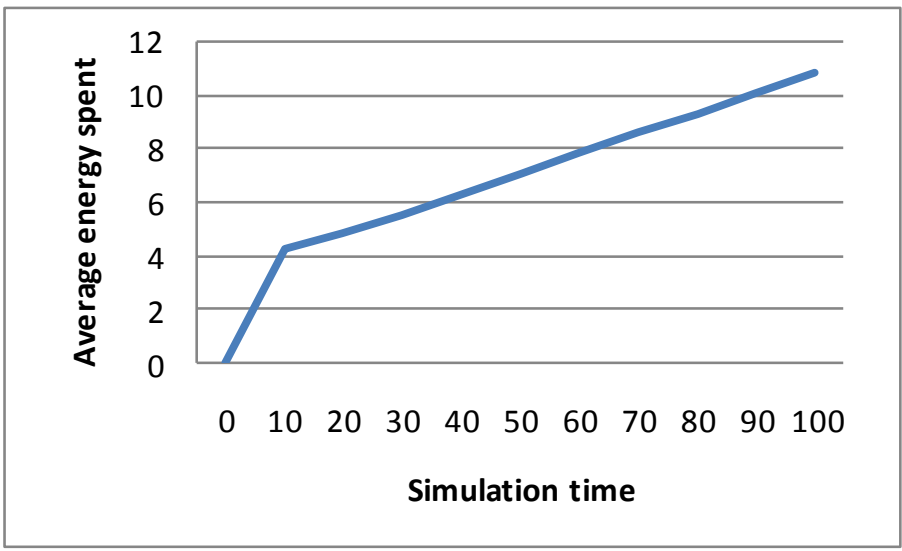

Fig. 3 shows average energy spent as the time increases, the average energy spent also increases.

For the second scenario we have focused on comparing the performance with regard to two metrics: average packet delivery rates and energy spent with respect to the no. of nodes.

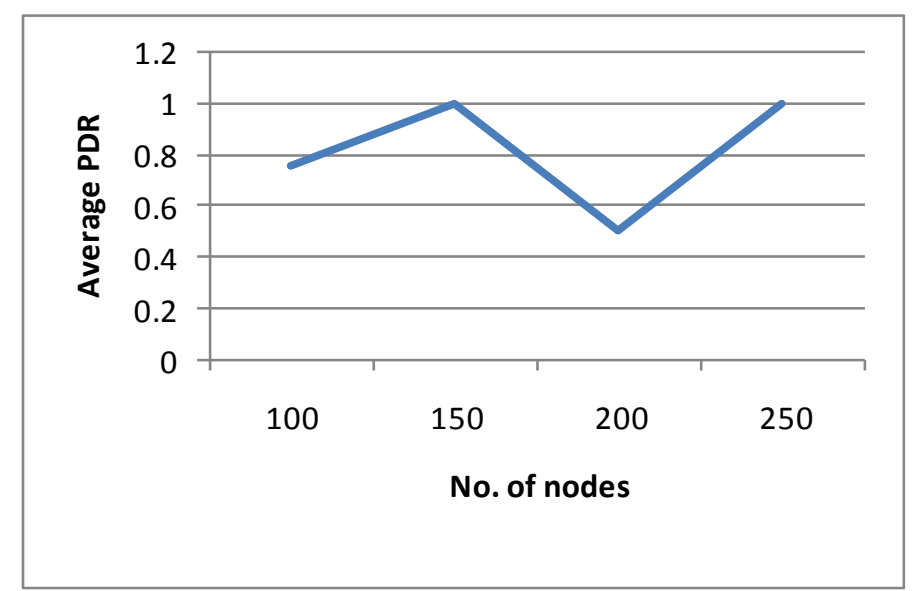

Fig. 4 shows average PDR as the no. of nodes increases. 


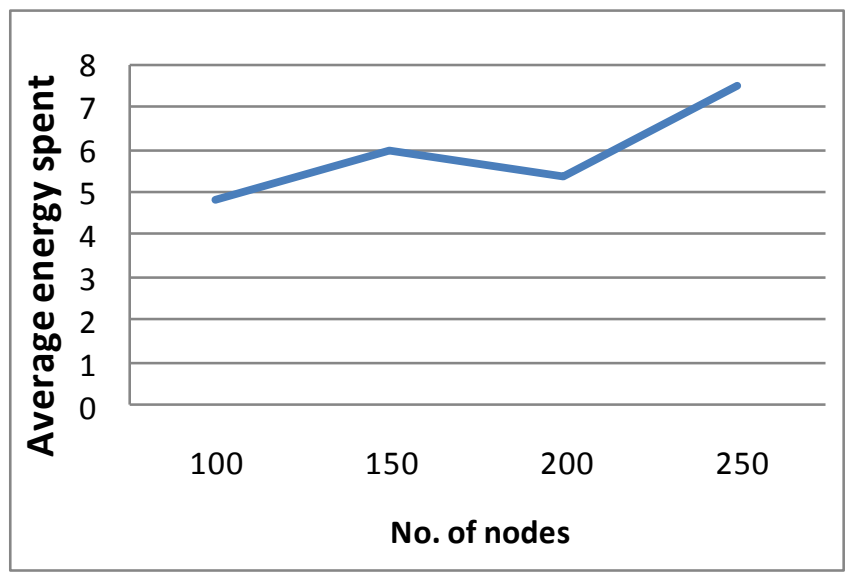

Fig. 5 shows average energy spent as the no. of nodes increases.

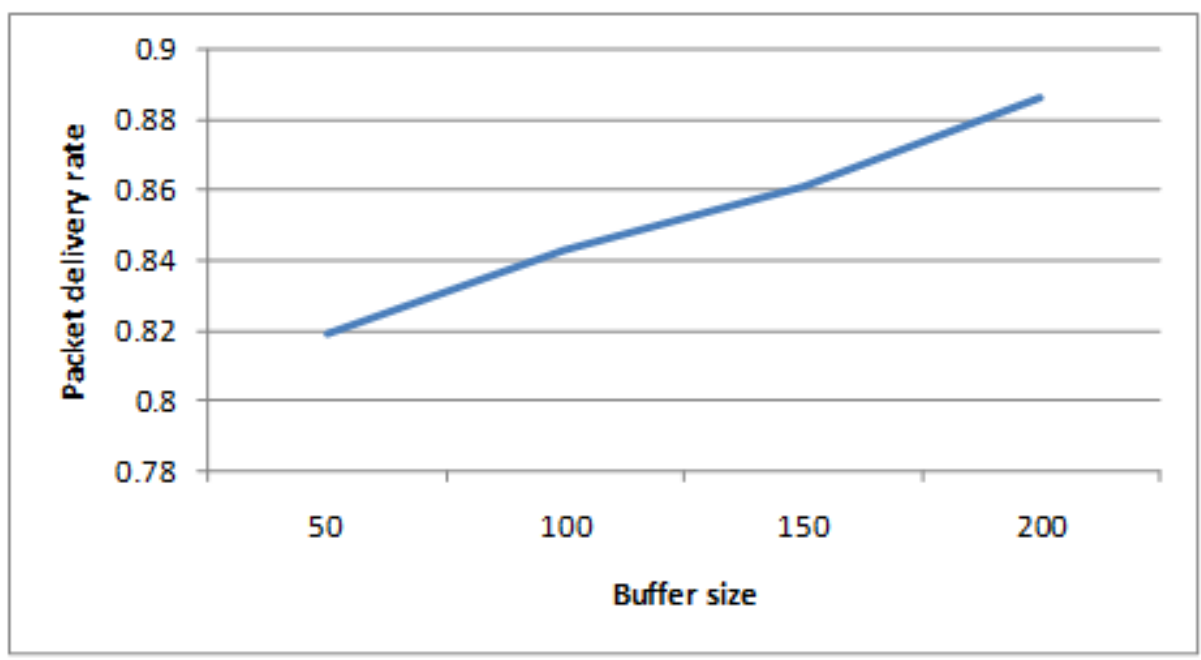

Fig. 6 shows packet delivery rate as the buffer size increases.

\section{Conclusion}

In this paper we analyzed the architecture of Delay tolerant network and implemented the PROPHET routing protocol. In DTN, as the name says due to opportunistic connections the time taken to deliver the message to the destination node is very high. As time increases more no. of packets get delivered because in PROPHET, rather than forwarding the message to all the encountered node, nodes with high delivery predictability is selected. So this procedure takes time and there is delay. In DTN, when a message has to reach to its destination, it goes via many intermediate nodes. These intermediate nodes sometimes have less energy value or less delivery predictability or less buffer size. Thus all intermediate nodes are not reliable for delivering or forwarding the message. In such case, if more no. of nodes performs in the routing process, the packet delivery rate will increase but on the other hand the average energy spent is also high. When a source node forward a message to an encountered node(with high delivery predictability) the message gets stored in the intermediate node's buffer. So if the node's buffer is small enough to receive a new message, it has to delete few of the oldest messages to get free buffer space. It is not sure whether the deleted messages is being delivered to the destinations or not. As time passes, most nodes' buffer are full so they have to discard many messages to receive a new message. This causes loss of many messages that have not been delivered to their destinations. So large buffer size is preferred. In Fig 6. It is seen that as the buffer size increases the PDR gets increased showing that large buffer size leads to more delivery of the message to their destination. 


\section{References}

[1] Kevin Fall, “A delay-tolerant network architecture for challenged internets”. Proceedings of ACM SIGCOMM. USA: ACM Press, 2003.

[2] V. Cerf, et al. , Network "Delay-Tolerant Networking Architecture",April 2007.

[3] Jahnabi Borah," Application of Computational Intelligence Paradigm in the Probabilistic Routing for intermittently connected network," IOSR Journal of Computer Engineering (IOSRJCEVolume 8, Issue 2, PP 32-36, Nov-Dec. 2012.

[4] Lindgren, et al., "Probabilistic Routing Protocol for Intermittently Connected Networks',,. Internet Research Task Force (IRTF), August 2012 .

[5] A. Lindgren, A. Doria, and O. Scheln, "Probabilistic Routing in Intermittently Connected Networks", in Proc. Workshop on Service Assurance with Partial and Intermittent Resources, August 2004.

[6] Jingfeng Xue, Jiansheng Li, Yuanda Cao, Ji Fang, “Advanced PROPHET Routing in Delay Tolerant Network” ,International Conference on Communication Software and Networks 2009.

[8] A. Vahdat and B. Becker, "Epidemic Routing for Partially-Connecte Ad- hoc Networks," Technical Report CS-2000-06, Duke University,July 2000.

[9] T. Spyropoulos, K. Psounis, and C. S. Raghavendra, "Spray and wait: Efficient routing in intermittently connected mobile networks," in Proceedings of ACMSIGCOMM workshop on Delay Tolerant Networking (WDTN), 2005.

\section{Authors Profile}

Jahnabi Borah received her B.Tech Degree in Information Technology from North Eastern Hill University, Shillong. Now she is an M.Tech student in the Department of Computer Science Engineering \& IT, Assam Don Bosco University, Assam. Her research interest includes Artificial Intelligence and Wireless Networks.

Dhruwajita Devi is working as Assistant Professor in the Department of Computer Science Engineering \& IT, Assam Don Bosco University, Assam.

Dr. Y. J Singh is working as Head \& Associate Professor, in the Department of Computer Science Engineering \& IT, Assam Don Bosco University, Assam . 